\title{
Fidaxomicin for Clostridium
} difficile-Associated Diarrhoea Epidemiological Method for Estimation of Warranted Price

\author{
David Alexander Sclar, ${ }^{1,2,3,4}$ Linda M. Robison, ${ }^{1,2}$ Ambartsum M. Oganov, ${ }^{1}$ \\ Jennifer M. Schmidt, ${ }^{1}$ Kurt A. Bowen ${ }^{1}$ and Leigh V. Castillo ${ }^{1}$
}

1 Pharmacoeconomics and Pharmacoepidemiology Research Unit, Washington State University, Spokane, WA, USA

2 Department of Health Policy and Administration, Washington State University, Spokane, WA, USA

3 Department of Pharmacotherapy, Washington State University, Spokane, WA, USA

4 Department of Statistics, Washington State University, Spokane, WA, USA

Abstract

Background: Fidaxomicin is a macrocyclic antibiotic approved in 2011 by the US Food and Drug Administration for treatment of Clostridium difficileassociated diarrhoea (CDAD).

Objective: Herein, we present an epidemiological method to estimate, on a case mix basis, and from the perspective of the US health system, the warranted (justifiable) price per day for fidaxomicin, as a percent of the wholesale acquisition cost (WAC) per day for fidaxomicin (\$US280).

Methods: Data from two randomized controlled studies (Optimer-003 $[\mathrm{n}=596]$ and Optimer-004 [ $\mathrm{n}=509])$ were used to discern the number-neededto-treat $(\mathrm{NNT}=7.1)$ for sustained clinical response. Sustained clinical response was defined as clinical response at the end of treatment, and survival without proven or suspected CDAD recurrence through 25 days beyond the end of treatment. National data for primary and secondary cases (the case mix) of CDAD (mean hospital length of stay [LOS], and mean cost) were derived from the 2009 US Healthcare Cost and Utilization Project. The method for attribution of hospital LOS for secondary cases of CDAD was derived from a study published by O'Brien et al. in 2007. Comparative regimens of vancomycin were: (i) injectable used orally, $125 \mathrm{mg}$ four times daily (qid; WAC of \$US6/day), with use of vancomycin hydrochloride $(\mathrm{HCl})$ capsules, $125 \mathrm{mg}$ qid (WAC of \$US106/day) post-hospital discharge; (ii) vancomycin $\mathrm{HCl}$ capsules, $125 \mathrm{mg}$ qid; and (iii) vancomycin $\mathrm{HCl}$ capsules, $250 \mathrm{mg}$ qid (WAC of \$US196/day). Findings are expressed in 2011 US dollars. The study perspective is that of the US health system.

Results: The warranted price per day for fidaxomicin represented $95 \%$ of the WAC per day for fidaxomicin compared with use of injectable vancomycin 
(orally) $125 \mathrm{mg}$ qid (with subsequent use of vancomycin $\mathrm{HCl}$ capsules, $125 \mathrm{mg}$ qid post-hospital discharge); $109 \%$ of the WAC per day for fidaxomicin compared with use of vancomycin $\mathrm{HCl}$ capsules, $125 \mathrm{mg}$ qid; and $141 \%$ of the WAC per day for fidaxomicin when compared with use of vancomycin $\mathrm{HCl}$ capsules, $250 \mathrm{mg}$ qid.

Conclusion: From the perspective of the US health system, fidaxomicin represents value for money in the treatment of CDAD. The methodology employed in this research has application beyond antimicrobial pharmacotherapy.

\section{Introduction}

Clostridium difficile is a Gram-positive, sporeforming, toxin-producing anaerobic bacillus. It is the most common cause of infectious diarrhoea in hospitalized patients in North America and Europe, where both the incidence and severity of the disease have increased significantly since 2000. ${ }^{[1]}$ Debate persists as to the effect of the emergence of a hyper-virulent $C$. difficile strain, denoted as NAP1/BI/027 (North American Pulsed Field type 1 [NAP1], restriction-endonuclease analysis [REA] type BI, or polymersase-chain-reaction biotype 027 ; collectively referred to as NAP1/ $\mathrm{BI} / 0270$ ), on the observed increase in both the incidence and severity of disease. ${ }^{[1]}$ That said, mortality due to $C$. difficile-associated diarrhoea (CDAD) has increased significantly over the past decade. ${ }^{[1,2]}$

At present it is estimated that NAP $1 / \mathrm{BI} / 027$ C. difficile is present in approximately one-third of cases. ${ }^{[1,2]}$ However, standard hospital laboratory procedure is to test for the presence of $C$. difficile toxin in a faecal sample prior to initiating pharmacotherapy; however, restriction-endonuclease strain typing is rarely, if ever, conducted. ${ }^{[3]}$

Guidelines published in 2010 from the Infectious Disease Society of America, and the Society for Healthcare Epidemiology of America, prioritize the usage of metronidazole or vancomycin for CDAD, depending upon disease severity. ${ }^{[4]}$

Fidaxomicin (Dificid ${ }^{\circledR}$, Optimer Pharmaceuticals, Inc., San Diego, CA, USA), a macrocyclic antibiotic approved in 2011 by the US Food and Drug Administration (FDA) for use in the treatment of $C$. difficile infection, is more active in vitro than vancomycin by a factor of approximately eight. ${ }^{[1]}$ Recent clinical trials indicate fidaxomicin is superior to vancomycin in terms of rate of recurrence of CDAD, and rate of global cure in patients infected with strains other than NAP1/BI/027 (i.e. Non-NAP1/BI/027). ${ }^{[1,5]}$

Internationally, payers are increasingly mandating the demonstration of value for money with regard to pharmacotherapy. ${ }^{[6]}$ Given the inability of clinicians to distinguish - for a given patient with infection - the strain of $C$. difficile responsible for the infection, as restriction-endonuclease strain typing is rarely, if ever, conducted, this inquiry utilized a novel epidemiological approach to estimate whether the market price for fidaxomicin is warranted (justifiable) from the perspective of the US health system.

\section{Methods}

The number-needed-to-treat (NNT), the reciprocal of the absolute-risk-reduction (ARR), is a practical evidence-based indicator denoting the number of persons requiring treatment (i.e. intervention) to prevent the occurrence of one undesirable (adverse) outcome, relative to that which would have been observed with use of an alternative (comparator) treatment, or standard care. ${ }^{[7]}$

Comparative data regarding the efficacy of fidaxomicin in the treatment of CDAD were derived from two pivotal randomized controlled trials (Optimer-003 [n=596], and Optimer-004 $[n=509]$, Optimer Pharmaceuticals, Inc.). ${ }^{[5]}$ The regimens employed in the two randomized clinical trials were 10 days' treatment with either fidaxomicin $200 \mathrm{mg}$ twice daily (bid; wholesale 
acquisition cost [WAC ${ }^{\mathbf{1}}$ ] of \$US280/day), or vancomycin hydrochloride $(\mathrm{HCl}) 125 \mathrm{mg}$ four times daily (qid; WAC of \$US106/day).

The pooled NNT for sustained clinical response was 7.1 (Wilson score method). ${ }^{[8]}$ Sustained clinical response was defined as clinical response at the end of treatment, and survival without proven or suspected CDAD recurrence through 25 days beyond the end of treatment. Based on the pooled NNT, for every seven patients ${ }^{2}$ treated with fidaxomicin, there was avoidance of one hospital readmission for CDAD, as compared with use of vancomycin.

In the present study, the comparative regimens of vancomycin were: (i) injectable administered orally, $125 \mathrm{mg}$ qid (WAC of \$US6/day); ${ }^{3}$ (ii) vancomycin $\mathrm{HCl}$ capsules, $125 \mathrm{mg}$ qid (WAC of \$US106/day); and (iii) vancomycin $\mathrm{HCl}$ capsules, $250 \mathrm{mg}$ qid (WAC of \$US196/day) [values are expressed in 2011 US dollars].

\section{Hospital Perspective}

National (US) data for primary and secondary cases of CDAD (International Classification of Diseases, 9th Revision, Clinical Modification [ICD-9-CM] code 008.45) were derived from the 2009 US Healthcare Cost and Utilization Project (HCUP). ${ }^{[9,10]}$ Data contained in each discharge summary pertinent to this study included patient demographics, expected source of primary payment, the diagnostic profile based on ICD-9-CM coding, measures of resource use, including mean hospital length of stay (LOS), and mean hospital cost, ${ }^{4}$ and disposition of the patient upon discharge, including mortality.

The attribution of hospital LOS and hospital cost in secondary cases of CDAD was based on the methodology advanced by O'Brien et al. ${ }^{[11]}$ Specifically, in secondary cases of CDAD, $18 \%$ of hospital LOS and $46 \%$ of hospital cost is attributable directly to CDAD. All findings stemming from this inquiry are expressed in 2011 dollars. ${ }^{5}$

For both primary and secondary cases of CDAD, and for each comparative regimen of vancomycin examined, the following equation was derived to discern, on a per-day basis, whether there existed a savings or loss (dollar value) net the market price (WAC) of fidaxomicin in the hospital (inpatient) environment:

$$
\pi_{1}=([((\lambda / \mathrm{NNT})+(\gamma \cdot \mathrm{LOS})) / \mathrm{LOS}]-\$ \mathrm{US} 280.00)
$$

where,

$\pi_{1}=$ estimate of savings or loss net the market price (WAC) for fidaxomicin on a per-day basis in the hospital;

$\lambda=$ mean hospital cost (2011 dollars) for either a primary or secondary case of CDAD;

LOS $=$ hospital LOS for a primary or secondary case of CDAD;

$\gamma=$ cost of vancomycin (alternative pharmacotherapy regimen) for a primary or secondary case of CDAD for the hospital LOS;

\$US280 = market price (WAC) of fidaxomicin on a per-day basis.

In this inquiry, it is assumed that the initiation of antimicrobial pharmacotherapy commenced on day one of the hospital LOS in all primary cases of CDAD, and for all of the attributable hospital LOS in secondary cases of CDAD. The aforementioned assumption established the most rigorous (conservative) threshold for the dependent variable $\pi_{1}$ in equation 1 to surmount (i.e. result in a savings [dollar value]).

\section{Ambulatory Perspective}

Upon hospital discharge, it is assumed patients with either a primary or a secondary case of CDAD were prescribed pharmacotherapy for the

1 WACs for pharmacotherapy referenced in this inquiry were obtained from First Data Bank.

2 This analysis utilized a pooled NNT and hospital length of stay rounded to an integer.

3 Assumed use of vancomycin $\mathrm{HCl}$ capsules, $125 \mathrm{mg}$ qid, for ambulatory duration of treatment.

4 Hospital costs, not charges, for ICD-9-CM code 008.45 were discerned from the 2009 HCUP Nationwide Inpatient Sample (NIS).

5 Inflation adjustment to 2011 dollars via US Bureau of Labor Statistics <http://www.bls.gov/data/inflation calculator.htm>. 
balance of a 10-day regimen, barring mortality during the LOS. For both primary and secondary cases of CDAD, and for each comparative regimen of vancomycin examined, the following equation was derived to discern, on a per-day basis, whether there existed a savings or loss (dollar value) with use of fidaxomicin in the ambulatory (outpatient) environment:

$$
\pi_{2}=(\gamma-\Phi)
$$

where,

$\pi_{2}=$ estimate of savings or loss for a primary or secondary case of CDAD, on a per-day basis, in the ambulatory environment;

$\gamma=$ cost of vancomycin (alternative pharmacotherapy regimen) for a primary or secondary case of CDAD on a per-day basis;

$\Phi=$ market price (WAC) of fidaxomicin for a primary or secondary case of CDAD on a perday basis.

\section{Health System Perspective}

For both primary and secondary cases of CDAD, and for each comparative regimen of vancomycin examined, the following equation was derived to discern whether there existed a savings or loss (dollar value) with use of fidaxomicin from the perspective of the US health system:

$$
\Pi=\pi_{3} \pm \pi_{4}
$$

where,

$\Pi=$ estimate of US health system savings or loss with use of fidaxomicin;

$\pi_{3}=$ total savings or loss, for a primary or secondary case of CDAD, based on days of pharmacotherapy in the hospital;

$\pi_{4}=$ total savings or loss, for a primary or secondary case of CDAD, based on days of pharmacotherapy in the ambulatory environment.

\section{Warranted Price for Fidaxomicin}

The following equation utilized the abovereferenced calculation of savings or loss (dollar value) at the hospital and ambulatory levels, for both primary and secondary cases of CDAD, and for each comparative regimen of vancomycin examined, to discern the warranted price of fi- daxomicin on a per-day basis:

$$
€=\$ U S 280 \pm \Delta
$$

where,

$€=$ warranted price for fidaxomicin at the hospital or ambulatory level;

$\$ U S 280=$ WAC per day for a regimen of fidaxomicin;

$\Delta=$ savings or loss (dollar value) at the hospital or ambulatory level, for both primary and secondary cases of CDAD, and for each comparative regimen of vancomycin examined, on a per-day basis.

In both primary and secondary cases of CDAD, the warranted price for fidaxomicin on a per-day basis, from the perspective of the US health system, is the weighted average of the warranted price per day, as discerned at the hospital and ambulatory levels, respectively, and for a given combination of hospital LOS and ambulatory duration. In turn, we report the warranted price per day for fidaxomicin, from the perspective of the US health system, as a percent of the WAC per day for fidaxomicin.

Finally, the warranted price per day for fidaxomicin, from the perspective of the US health system, as a percent of the WAC per day for fidaxomicin, in both primary and secondary cases of CDAD, is weighted by the case mix for CDAD (primary or secondary) in 2009, in order to yield an aggregate appraisal (from the perspective of the US health system) of the warranted price per day for fidaxomicin, as a percent of the WAC per day for fidaxomicin.

\section{Results}

In 2009, there were 336565 hospitalizations wherein CDAD (ICD-9-CM code 008.45) was recorded in the discharge summary, with 110553 discharge summaries reporting CDAD as the primary diagnosis. For the 336565 hospitalizations wherein CDAD was recorded in the discharge summary, $65 \%$ were $>65$ years of age, $57 \%$ were female, and $68 \%$ of discharges were paid by Medicare as the primary insurance carrier. Of the 110553 hospitalizations wherein CDAD was recorded in the discharge summary as the pri- 
mary diagnosis, $67 \%$ were $>65$ years of age, $64 \%$ were female, $69 \%$ of discharges were paid by Medicare as the primary insurance carrier, and the mortality rate was $3.7 \%$. Of the 226408 hospitalizations wherein CDAD was recorded in the discharge summary as a secondary diagnosis, $64 \%$ were $>65$ years of age, $54 \%$ were female, $67 \%$ of discharges were paid by Medicare as the primary insurance carrier, and the mortality rate was $11.7 \% .^{6}$

The mean \pm standard error (SE) hospital LOS for a primary case of CDAD in 2009 was 6.9 days \pm 0.1 , with a mean hospital cost of \$US10 644 (2011 dollars). The mean hospital LOS for a patient with a secondary case of CDAD was 15.9 days \pm 0.1 , with an attributed LOS due to CDAD of 2.9 days. The mean hospital cost for a patient with a secondary case of CDAD was \$US34 260, with an attributed hospital cost due to CDAD of SUS15 759.

Table I presents the savings or loss (dollar value) per day with use of fidaxomicin as compared with vancomycin for primary cases of CDAD, the warranted price per day for fidaxomicin from a hospital, ambulatory and health system perspective, and the warranted price per day for fidaxomicin, from the perspective of the US health system, as a percent of the WAC per day for fidaxomicin. Results indicate that the warranted price per day for fidaxomicin, from the perspective of the US health system, represents $67 \%$ of the WAC per day for fidaxomicin when compared with use of injectable vancomycin $125 \mathrm{mg}$ qid administered orally (with subsequent use of vancomycin $\mathrm{HCl}$ capsules, $125 \mathrm{mg}$ qid in the ambulatory environment), $92 \%$ of the WAC per day for fidaxomicin when compared with use of vancomycin $\mathrm{HCl}$ capsules, $125 \mathrm{mg}$ qid, and $124 \%$ of the WAC per day for fidaxomicin when compared with use of vancomycin $\mathrm{HCl}$ capsules, $250 \mathrm{mg}$ qid.

The savings or loss (dollar value) per day with use of fidaxomicin as compared with vancomycin
Table I. Primary cases of Clostridium difficile-associated diarrhoea (CDAD): savings or loss per day (\$US) [hospital and ambulatory], and in total (US health system) with use of fidaxomicin (FDX) as compared with vancomycin, warranted price per day for FDX from a hospital, ambulatory and US health system perspective, and the warranted price per day for FDX, from the perspective of the US health system, as a percent of the wholesale acquisition cost (WAC) per day for FDX

\begin{tabular}{llll}
\hline $\begin{array}{l}\text { Primary cases } \\
\text { of CDAD }\end{array}$ & \multicolumn{3}{l}{ Comparative regimen of vancomycin } \\
\cline { 2 - 4 } & $\begin{array}{l}\text { Injectable } \\
125 \mathrm{mg} \text { qid } \\
\text { (oral admin.) }\end{array}$ & $\begin{array}{l}\text { Capsules } \\
125 \mathrm{mg} \text { qid }\end{array}$ & $\begin{array}{l}\text { Capsules } \\
250 \mathrm{mg} \text { qid }\end{array}$ \\
\hline Savings or loss by perspective & & \\
Hospital (7 days) & $-56.37 /$ day & $+43.63 /$ day & $+133.63 /$ day \\
Ambulatory (3 days) & $-174.00 /$ day & $-174.00 /$ day & $-84.00 /$ day \\
Health system & $-916.57 /$ day & $-216.57 /$ day & $+683.43 /$ day \\
Warranted price for FDX by perspective & \\
Hospital (7 days) & $+223.63 /$ day & $+323.63 /$ day & $+414.63 /$ day \\
Ambulatory (3 days) & $+106.00 /$ day & $+106.00 /$ day & $+196.00 /$ day \\
Health system & $+188.34 /$ day & $+258.34 /$ day & $+348.34 /$ day \\
Percent of WAC & 67 & 92 & 124
\end{tabular}

a Cost of comparative regimens of vancomycin: (i) injectable used orally, $125 \mathrm{mg}$ qid (WAC of \$US6/day); (ii) vancomycin hydrochloride $(\mathrm{HCl}$ ) capsules, $125 \mathrm{mg}$ qid (WAC of $\$$ US106/day); and (iii) vancomycin $\mathrm{HCl}$ capsules, $250 \mathrm{mg}$ qid (WAC of $\$ U S 196 /$ day).

b Assumed use of vancomycin capsules, $125 \mathrm{mg}$ qid, for ambulatory duration of treatment.

c Cost of fidaxomicin: $200 \mathrm{mg}$ bid (WAC of $\$$ US280/day).

admin. = administration; bid = twice daily; qid = four times daily.

for secondary cases of CDAD, the warranted price per day for fidaxomicin from a hospital, ambulatory and health system perspective, and the warranted price per day for fidaxomicin, from the perspective of the US health system, as a percent of the WAC per day for fidaxomicin are presented in table II. Results indicate that the warranted price per day for fidaxomicin, from the perspective of the US health system, represents $108 \%$ of the WAC per day for fidaxomicin when compared with use of injectable vancomycin $125 \mathrm{mg}$ qid administered orally (with subsequent use of vancomycin $\mathrm{HCl}$ capsules, $125 \mathrm{mg}$ qid in the ambulatory environment), $118 \%$ of the WAC per day for fidaxomicin when compared with use

6 Primary and secondary cases of CDAD (ICD-9-CM code 008.45) do not add to the aggregate number of CDAD cases reported in the US in 2009, due to weightings employed in the HCUP NIS, and the resulting standard error (SE) for each mean. Estimated percentage of primary cases $=32.8 \%$; estimated percentage of secondary cases $=67.2 \%$. 
of vancomycin $\mathrm{HCl}$ capsules, $125 \mathrm{mg}$ qid, and $150 \%$ of the WAC per day for fidaxomicin when compared with use of vancomycin $\mathrm{HCl}$ capsules, $250 \mathrm{mg}$ qid.

Table III details the warranted price per day for fidaxomicin, from the perspective of the US health system, as a percent of the WAC per day for fidaxomicin, having accounted for the case mix for CDAD (primary or secondary) in 2009. Results indicate that on a case mix basis, the warranted price per day for fidaxomicin, from the perspective of the US health system, represents 95\% of the WAC per day for fidaxomicin when compared with use of injectable vancomycin $125 \mathrm{mg}$ qid administered orally (with subsequent use of vancomycin $\mathrm{HCl}$ capsules, $125 \mathrm{mg}$ qid in the ambulatory environment), $109 \%$ of the WAC per day for fidaxomicin when compared with use of vancomycin $\mathrm{HCl}$ capsules, $125 \mathrm{mg}$ qid, and $141 \%$ of the WAC per day for fidaxomicin when

Table II. Secondary cases of Clostridium difficile-associated diarrhoea (CDAD): savings or loss per day (\$US) [hospital and ambulatory], and in total (US health system) with use of fidaxomicin (FDX) as compared with vancomycin, warranted price per day for FDX from a hospital, ambulatory and US health system perspective, and the warranted price per day for FDX, from the perspective of the US health system, as a percent of the wholesale acquisition cost (WAC) per day for FDX

\begin{tabular}{llll}
\hline Secondary cases & \multicolumn{3}{l}{ Comparative regimen of vancomycin ${ }^{\mathrm{a}}$} \\
\cline { 2 - 4 } of CDAD & $\begin{array}{l}\text { Injectable } \\
\text { 125 mg qid }\end{array}$ & Capsules & Capsules \\
& (oral admin.) & & \\
& & & \\
\hline
\end{tabular}

\begin{tabular}{llll}
\hline Savings or loss by perspective & & \\
Hospital (3 days) & $+476.43 /$ day & $+576.43 /$ day & $+666.43 /$ day \\
Ambulatory $(7$ days $)$ & $-174.00 /$ day $^{\text {b }}$ & $-174.00 /$ day & $-84.00 /$ day \\
Health system & $+211.29 /$ day & $+511.29 /$ day & $+1411.29 /$ day \\
Warranted price for fidaxomicin by perspective & \\
Hospital (3 days) & $+756.43 /$ day & $+856.43 /$ day & $+946.43 /$ day \\
Ambulatory $(7$ days $)$ & $+106.00 /$ day & $+106.00 /$ day & $+196.00 /$ day \\
Health system & $+301.13 /$ day & $+331.13 /$ day & $+421.13 /$ day \\
Percent of WAC & 108 & 118 & 150
\end{tabular}

a Cost of comparative regimens of vancomycin: (i) injectable used orally, $125 \mathrm{mg}$ qid (WAC of \$US6/day); (ii) vancomycin hydrochloride $(\mathrm{HCl})$ capsules, $125 \mathrm{mg}$ qid (WAC of $\$$ US106/day); and (iii) vancomycin $\mathrm{HCl}$ capsules, $250 \mathrm{mg}$ qid (WAC of $\$ U S 196 /$ day).

b Assumed use of vancomycin capsules, $125 \mathrm{mg}$ qid, for ambulatory duration of treatment.

c Cost of FDX: $200 \mathrm{mg}$ bid (WAC of $\$ U S 280 /$ day).

admin. = administration; bid = twice daily; qid = four times daily.
Table III. Primary and secondary cases of Clostridium difficileassociated diarrhoea (CDAD): the warranted price per day (\$US) for fidaxomicin (FDX), from the perspective of the US health system, as a percent of the wholesale acquisition cost (WAC) per day for FDX, ${ }^{a}$ weighted by the US case mix (primary and secondary) for CDAD in $2009^{\mathrm{b}}$

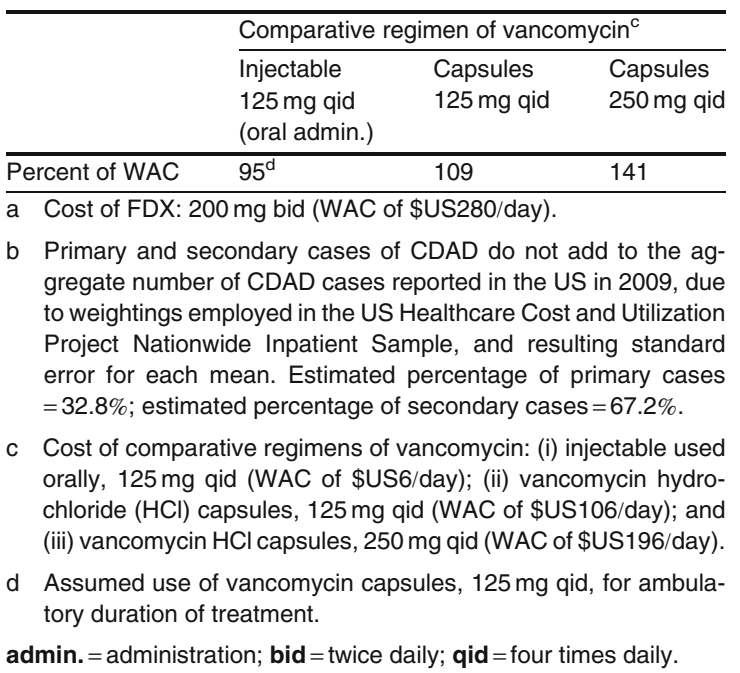

compared with use of vancomycin $\mathrm{HCl}$ capsules, $250 \mathrm{mg}$ qid.

\section{Discussion}

Comparative effectiveness research (CER), as defined by the US Agency for Healthcare Research and Quality (AHRQ), is to be designed to inform stakeholders of the clinical and/or economic benefit(s) of competing alternatives. ${ }^{[12]}$ This inquiry utilized a novel epidemiological method to estimate, on a case mix basis, and from the perspective of the US health system, the warranted price per day for fidaxomicin, as a percent of the WAC per day for fidaxomicin. Findings ranged from $95 \%$ to $141 \%$ across the alternative regimens of vancomycin examined. These results, coupled with recent evidence indicating fidaxomicin is significantly more effective than vancomycin in achieving clinical cure for CDAD in the presence of concomitant antimicrobial pharmacotherapy, and in preventing recurrence of disease, underscores the clinical and economic benefits to be ascribed to fidaxomicin. ${ }^{[13]}$ 
We note that among primary cases of CDAD, the warranted price per day for fidaxomicin, from the perspective of the US health system, represents $67 \%$ of the WAC per day for fidaxomicin when compared with use of injectable vancomycin $125 \mathrm{mg}$ qid administered orally (with subsequent use of vancomycin $\mathrm{HCl}$ capsules, $125 \mathrm{mg}$ qid in the ambulatory environment). We hypothesize that the majority of primary cases of CDAD are due to recurrence of disease, and that use of fidaxomicin will reduce the proportion of primary cases annually. Thus, there exists the potential for the warranted price per day for fidaxomicin, from the perspective of the US health system, to increase from the present estimate of $95 \%$ of the WAC per day for fidaxomicin to that of parity (i.e. $\geq 100 \%$ of the WAC per day for fidaxomicin) when compared with use of injectable vancomycin $125 \mathrm{mg}$ qid administered orally (with subsequent use of vancomycin $\mathrm{HCl}$ capsules, $125 \mathrm{mg}$ qid, in the ambulatory environment).

Health service substitution effects have been observed across the components of the US health system, and in the treatment of a wide range of disease states. ${ }^{[14,15]}$ Our findings indicate that for the ambulatory (outpatient) duration of the treatment regimen, use of fidaxomicin results in a loss (dollar value) on a per-day basis relative to each of the comparative regimens of vancomycin examined, and that the warranted price per day for fidaxomicin, as a percent of the WAC per day for fidaxomicin, was far less than the threshold value of \$US280 per day. However, in the majority of primary-case scenarios, and in all secondary-case scenarios of CDAD examined, use of fidaxomicin during the hospital duration of the treatment regimen resulted in a savings (dollar value) on a per-day basis relative to each of the comparative regimens of vancomycin examined, and the warranted price per day for fidaxomicin exceeded the threshold value of \$US280 per day. Thus, the offset potential between the hospital and ambulatory components of care were observed in the findings for the US health system. Stakeholders need to recognize that a loss (dollar value) - from an accounting perspective - in one component of the US health system may not connote a loss to the enterprise in the aggregate.
This research is constrained by the nature of the US HCUP NIS, as data are not collected as to the day pharmacotherapy is initiated within a given hospital LOS. That said, our assumption that the initiation of antimicrobial pharmacotherapy commenced on day one of the hospital LOS in all primary cases of CDAD, and for all of the attributable hospital LOS in secondary cases of CDAD, established the most rigorous (conservative) threshold for the dependent variable $\pi_{1}$ in equation 1 to surmount (i.e. result in a savings [dollar value]). Finally, as restriction-endonuclease strain typing is rarely, if ever, conducted, we were unable to account for the magnitude of the incidence of the hyper-virulent $C$. difficile strain $\mathrm{NAP} 1 / \mathrm{BI} / 027$, and the resulting effect on the efficacy of fidaxomicin.

\section{Conclusion}

The incidence, severity, mortality and expenditures associated with CDAD have increased significantly over the past decade. From the perspective of the US health system, fidaxomicin represents value for money in the treatment of CDAD. Future prospective research is required to confirm the findings presented herein. The epidemiologically-based method employed in this research has application beyond the evaluation of antimicrobial pharmacotherapy for the treatment of CDAD.

\section{Acknowledgements}

This study was supported by Optimer Pharmaceuticals, Inc. It was presented at the 46th Annual Midyear Clinical Meeting of the American Society of Health System Pharmacists, New Orleans, LA, USA, 6 December 2011.

Author disclosure: Dr Sclar and Ms Robison have served as consultants and received research support from Optimer Pharmaceuticals, Inc.

Author contributions: Conceptualization and design: Dr Sclar, Ms Robison, Mr Oganov, Ms Schmidt, Mr Bowen and Mr Castillo.

Data analysis and interpretation: Dr Sclar, Ms Robison, Mr Oganov, Ms Schmidt, Mr Bowen and Mr Castillo.

Manuscript development and revision: Dr Sclar, Ms Robison, Mr Oganov, Ms Schmidt, Mr Bowen and Mr Castillo.

Mr Oganov, Ms Schmidt, Mr Bowen and Mr Castillo are Pharm.D. Candidates, College of Pharmacy, Washington State University, Spokane, WA, USA. 


\section{References}

1. Louie TJ, Miller MA, Mullane KM, et al. FDX versus vancomycin for $C$. difficile infection. New Eng J Med 2011; 364: 422-31

2. Miller M, Gravel D, Mulvey M, et al. Health care-associated C. difficile infection in Canada: patient age and infecting strain type are highly predictive of severe outcome and mortality. Clin Infect Dis 2010; 50: 194-201

3. Killgore G, Thompson A, Johnson S, et al. Comparison of seven techniques for typing international epidemic strains of Clostridium difficile. J Clin Microbiol 2008; 46: 431-7

4. Cohen SH, Gerding DN, Johnson S, et al. Clinical practice guidelines for Clostridium difficile infection in adults: 2010 update by the society for healthcare epidemiology of America (SHEA) and the infectious diseases society of America (IDSA). Infect Control Hosp Epidemiol 2010; 31 : 431-55

5. Dificid ${ }^{\circledR}$ [package insert]. San Diego (CA): Optimer Pharmaceuticals, August, 2011

6. Neumann PJ. Evidence-based and value-based formulary guidelines. Health Aff (Millwood) 2004; 23: 124-34

7. Replogle WH, Johnson WD. Interpretation of absolute measures of disease risk in comparative research. Fam Med 2007; 39: 432-5

8. Newcombe RG. Interval estimation for the difference between independent proportions: comparison of eleven methods. Stat Med 1998; 17: 873-90

9. U.S. Public Health Service and Health Care Financing Administration. The International Classification of Diseases, 9th Rev., Clinical Modification. Volume 2.(DHHS Pub. No. (PHS) 89-1260). Washington, DC: Public Health Service, 1989

10. U.S. Nationwide Inpatient Sample (NIS). U.S. Healthcare Cost and Utilization Project (HCUP). Rockville (MD): Agency for Healthcare Research and Quality, 2009
11. O'Brien JA, Lahue BJ, Caro JJ, et al. The emerging infectious challenge of Clostridium difficile-associated disease in Massachusetts hospitals: clinical and economic consequences. Infect Control Hosp Epidemiol 2007; 28: 1219-27

12. Chang SM. The Agency for Healthcare Research and Quality (AHRQ) Effective Health Care (EHC) Program Methods Guide for Comparative Effectiveness Reviews: keeping up-to-date in a rapidly evolving field. J Clin Epidemiol 2011; 64: 1166-7

13. Mullane KM, Miller MA, Weiss K, et al. Efficacy of fidaxomicin versus vancomycin as therapy for Clostridium difficile infection in individuals taking concomitant antibiotics for other concurrent infections. Clin Infect Dis 2011; 53: 440-7

14. Kozma CM, Reeder CE, Lingle EW. Expanding Medicaid drug formulary coverage. Effects on utilization of related services. Med Care 1990; 28: 963-77

15. Sclar DA, Evans MA, Skaer TL, et al. Economic and epidemiologic modeling of full-length antihemophilic factor (recombinant), plasma/albumin-free method (rAHF-PFM), in previously treated patients with hemophilia A: comparison with $\mathrm{B}$-domain deleted rFVIII (BDDrFVIII), and utility of potential viral transmission reduction due to plasma/albumin-free status. Drugs R D 2005; 6: 149-56

Correspondence: Dr David Alexander Sclar, B.Pharm., Ph.D., Boeing Distinguished Professor of Health Policy \& Administration, Director, Pharmacoeconomics and Pharmacoepidemiology Research Unit, Department of Health Policy and Administration, College of Pharmacy, Washington State University, P.O. Box 1495, Spokane, WA 99210-1495, USA.

E-mail: sclar@mail.wsu.edu 